\title{
Controlabilidad Exacta de la Ecuación del Telégrafo Generalizada
}

\author{
J.A. SORIANO
}

ABSTRACT. In this paper we present a study of the exact controllability, in the boundary, of the generalized telegraph equation

$$
\left(K_{1}(x, t) y^{\prime}\right)^{\prime}+K_{2}(x, t) y^{\prime}+K_{3}(x, t) y-\sum_{i, j=1}^{n} \frac{\partial}{\partial x_{i}}\left(a_{i j}(x, t) \frac{\partial y}{\partial x_{j}}\right)=0 \text { en } Q,
$$

where $Q$ is the finite cylinder $\Omega \times] 0, T$ [ and $\Omega$ is a bounded domain in $\mathbf{R}^{\mathbf{n}}$. The result is obtained by the HUM (Hilbert Uniqueness Method) method introduced by J.L. Lions [8] and [9].

\section{INTRODUCCION}

Sea $\Omega$ un dominio acotado de $\mathbf{R}^{\mathrm{n}}$ con frontera $\Gamma$ de clase $C^{2}$ y $Q$ el cilindro finito $\Omega \times] 0, T$ [ con frontera lateral $\Sigma=\Gamma \times] 0, T$. Consideremos el sistema $\left(^{*}\right)$ que sigue con condiciones de contorno no homogéneas:

1991 Mathematica Subject Classification:

Servicio publicaciones Univ. Complutense. Madrid, 1995. 


$$
\left\{\begin{array}{l}
\left(K_{1}(x, t) y^{\prime}\right)^{\prime}+K_{2}(x, t) y^{\prime}+K_{3}(x, t) y \\
-\sum_{i, j=1}^{n} \frac{\partial}{\partial x_{i}}\left(a_{i j}(x, t) \frac{\partial y}{\partial x_{j}}\right)=0 \text { en } Q \\
y=v \text { sobre } \Sigma \\
y(0)=y^{0}, y^{\prime}(0)=y^{\prime} \text { en } \Omega
\end{array}\right.
$$

El problema de la controlabilidad exacta para el sistema $\left({ }^{*}\right)$ se formula de la siguiente forma: "Dado $T>0$ suficientemente grande, es posible, para cada par de datos iniciales en un espacio apropiado sobre $\Omega$, hallar un correspondiente control $v$, tal que la solucion $y=y(x, t)$ de (*), verifique la condición de reposo

$$
y(T)=0, y^{\prime}(T)=0 . "
$$

El sistema $\left(^{*}\right)$ es una generalización de la ecuación del telégrafo unidimensional:

$$
C L y^{\prime \prime}+(C R+G L) y^{\prime}+G R y-y_{x x}=0
$$

en donde $C, G, L$ y $R$ son los coeficientes de capacidad, pérdida, autoinducción y resistencia, respectivamente, calculados para la unidad de longitud, cf. A.N. Tijonov-A.A. Samarsky [19].

En este trabajo probaremos que el sistema $\left({ }^{*}\right)$ es exactamente controlable. Para tal usamos el método HUM (Hilbert Uniqueness Method) propuesto por J.L. Lions [8] y [9]. esto es posible, gracias a que en nuestro caso tenemos unicidad, reversibilidad y regularidad de soluciones.

Interesado en la controlabilidad exacta para el sistema $\left({ }^{*}\right)$ notamos que el caso $K_{1}(x, t)=1, K_{2}(x, t)=K_{3}(x, t)=0$ y $a_{i j}(x, t)=\delta_{i j} a(t)$ fue estudiado por J.L. Lions [10] y J.E. Muñoz Rivera [17], y el caso $K_{1}(x, t)=1, K_{2}(x, t)=K_{3}(x, t)=0$ y $a_{i j}(x, t)=\delta_{i j} a(x)$, por $\mathrm{E}$. Zuazua [21]. Seguidamente R. Fuentes [6] analizó la situación $K_{1}(x, t)=$ $1, K_{2}(x, t)=K_{3}(x, t)=0$. Nuestro análisis es diferente al de los autores mencionados arriba y los incluye.

Diversos autores usaron el método HUM en el estudio de la controlabilidad exacta de sistemas distribuidos, entre ellos podemos mencionar: 
L.A. Medeiros [14], L.A. Medeiros-R. Fuentes [13], C. Fabre-J.P. Puel [5], E. Zuazua [20], [21], C. Fabre [4], V. Komornik [7] y M. Milla Miranda [15], [16].

\section{RESULTADO PRINCIPAL}

Primero introduciremos algunas notaciones (cf. J.L. Lions [9]). Sean $x^{0} \in \mathbb{R}^{\mathrm{n}}, m(x)$ la función $x-x^{0}$ y $\nu(x)$ el vector normal unitario en $x \in \Gamma$, dirigida hacia el exterior de $\Omega$. Consideremos:

$$
R\left(x^{0}\right)=\max _{x \in \bar{\Omega}}\|m(x)\|=\max _{x \in \bar{\Omega}}\left|\sum_{l=1}^{n}\left(x_{l}-x_{l}^{0}\right)^{2}\right|^{1 / 2}
$$

Dividimos la frontera $\Gamma$ de $\Omega$ en dos partes:

$$
\begin{aligned}
& \Gamma\left(x^{0}\right)=\{x \in \Gamma: m(x) \cdot \nu(x)>0\} \\
& \Gamma_{*}\left(x^{0}\right)=\{x \in \Gamma: m(x) \cdot \nu(x) \leq 0\}=\Gamma \backslash \Gamma\left(x^{0}\right) ;
\end{aligned}
$$

así como la frontera lateral $\Sigma$ :

$$
\begin{aligned}
& \left.\Sigma\left(x^{0}\right)=\Gamma\left(x^{0}\right) \times\right] 0, T[ \\
& \left.\Sigma_{*}\left(x^{0}\right)=\Gamma_{*}\left(x^{0}\right) \times\right] 0, T\left[=\Sigma \backslash \Sigma\left(x^{0}\right) .\right.
\end{aligned}
$$

Representemos por $\lambda_{1}$ el primer autovalor del problema espectral $-\Delta \varphi=\lambda \varphi, \varphi \in H_{0}^{1}(\Omega)$.

Sean $K_{1}, K_{2}, K_{3}, a_{i j}: \mathbb{R}^{\mathrm{n}} \times[0, \infty[\rightarrow] 0, \infty[$ funciones contínuas.

Consideremos las siguientes hipótesis.

$$
\left\{\begin{array}{l}
K(x, t) \geq \alpha_{0}>0, \forall t \in[0, T] \text { c.t.p. en } \Omega \\
K_{1} \in C\left([0, T] ; W^{1, \infty}(\Omega)\right) \cap W^{2, \infty}\left(0, T ; L^{\infty}(\Omega)\right) \\
K_{1}^{\prime} \in L^{1}\left(0,+\infty, L^{\infty}(\Omega)\right) \\
\frac{\partial K_{1}}{\partial x_{\ell}} \in L^{1}\left(0,+\infty, L^{\infty}(\Omega)\right), 1 \leq \ell \leq n
\end{array}\right.
$$




$$
\begin{aligned}
& \left\{\begin{array}{l}
K_{2} \in L^{\infty}\left(0, T ; W^{1, \infty}(\Omega)\right) \cap W^{2, \infty}\left(0, T ; L^{\infty}(\Omega)\right) \\
K_{2} \in W^{1,1}\left(0,+\infty, L^{\infty}(\Omega)\right)
\end{array}\right. \\
& \left\{\begin{array}{c}
K_{3} \in W^{1, \infty}\left(0, T ; L^{\infty}(\Omega)\right) \\
K_{3} \in L^{1}\left(0,+\infty, L^{\infty}(\Omega)\right)
\end{array}\right.
\end{aligned}
$$

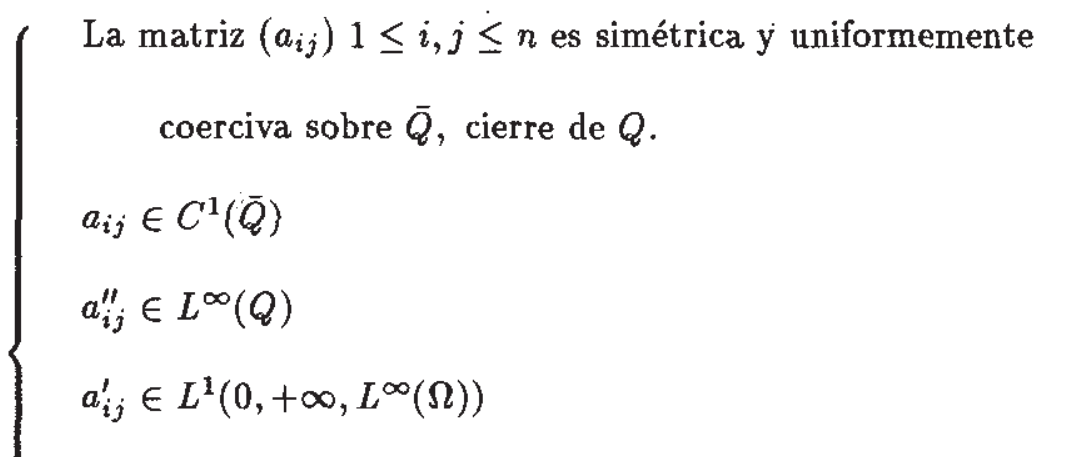

Existe $\delta>0$ tal que

$$
\begin{gathered}
(1-\delta) a_{i j}(x, t) \xi_{i} \xi_{j}-\frac{1}{2} \frac{\partial a_{i_{j}}(x, t)}{\partial x_{\ell}} m_{\ell} \xi_{i} \xi_{j} \geq 0 \\
\forall\{x, t\} \in Q \text { e } \xi \in \mathbb{R}^{\mathbf{n}}
\end{gathered}
$$

(Aqui y en lo que sigue adoptamos el convenio que dos índices repetidos indicarán sumación).

Deseamos actuar solmente sobre una parte de la frontera $\Sigma$, lo que formularemos como sigue.

Consideremos el siguiente sistema: 


$$
\left\{\begin{array}{l}
\left(K_{1}(x, t) y^{\prime}\right)^{\prime}+K_{2}(x, t) y^{\prime}+K_{3}(x, t) y+A(t) y=0 \text { en } Q \\
y=\left\{\begin{array}{l}
v \text { sobre } \Sigma\left(x^{0}\right) \\
0 \text { sobre } \Sigma \backslash \Sigma\left(x^{0}\right)
\end{array}\right. \\
y(0)=y^{0}, y^{\prime}(0)=y^{1} \text { en } \Omega
\end{array}\right.
$$

donde

$$
A(t) y=-\frac{\partial}{\partial x_{i}}\left(a_{i_{j}}(x, t) \frac{\partial y}{\partial x_{j}}\right)
$$

En (4.17) daremos un tiempo especial $T_{0}$ dependiendo de $n, R\left(x^{0}\right)$, $\lambda_{1}, \alpha_{0}, a_{0}, \delta$, las funciones $K_{1}, K_{2}, K_{3}, a_{i j}$ y sobre la geometría de $\Omega$.

Ahora enunciaremos el resultado principal de este trabajo.

Teorema 2.1. Supongamos que las hipótesis (H1)-(H4) sean satisfechas. Sea $T>T_{0}$. Entonces, para cada dato inicial $\left\{y^{0}, y^{1}\right\}$ perteneciendo a $L^{2}(\Omega) \times H^{-1}(\Omega)$, existe un control $v \in L^{2}\left(\Sigma\left(x^{0}\right)\right)$ tal que la solución ultradébil $y=y(x, t)$ del problema satisface

$$
y(T)=0, \quad y^{\prime}(T)=0 .
$$

La prueba del teorema 2.1 será dada en las tres secciones siguientes.

\section{EL PROBLEMA HOMOGENEO}

Introduciremos algunas notaciones que usaremos a lo largo del trabajo. Con $(, \cdot),,|\cdot|$ denotaremos el producto escalar y la norma de $L^{2}(\Omega)$ y con $\|\cdot\|$, la norma de $H_{0}^{1}(\Omega)$ dada por la forma de Dirichlet. La dualidad entre el espacio $F$ y su dual $F^{\prime}$ será denotada por $(\cdot, \cdot)$.

En esta sección obtendremos la existencia y la identidad de la energía para soluciones del siguiente sistema homogéneo: 


$$
\left\{\begin{array}{l}
\left(K_{1}(x, t) \phi^{\prime}\right)^{\prime}-\left(K_{2}(x, t) \phi\right)^{\prime}+K_{3}(x, t) \phi+A(t) \phi=f \text { en } Q \\
\phi=0 \text { sobre } \Sigma \\
\phi(0)=\phi^{0}, \phi^{\prime}(0)=\phi^{1} \text { en } \Omega .
\end{array}\right.
$$

Con dato $\left\{\phi^{\prime}, \phi^{1}, f\right\}$ en $H_{0}^{1}\left(\Omega \times L^{2}(\Omega) \times L^{1}\left(0, T ; L^{2}(\Omega)\right)\right.$. LLamaremos solución débil del problema (3.1) a la función $\phi: Q \rightarrow \mathbb{R}$, si $\phi$ pertenece a la clase

$$
\phi \in L^{\infty}\left(0, T ; H_{0}^{1}(\Omega)\right), \quad \phi^{\prime} \in L^{\infty}\left(0, T ; L^{2}(\Omega)\right)
$$

satisface la ecuación

$$
\frac{d}{d t}\left(K_{1} \phi^{\prime}, w\right)-\left(\left(K_{2} \phi\right)^{\prime}, w\right)+\left(K_{3} \phi, w\right)+a(t, \phi, w)=(f, w)
$$

en el sentido de $\mathcal{D}^{\prime}(0, T), \forall w \in H_{0}^{1}(\Omega)$ y las condiciones iniciales

$$
\phi(0)=\phi^{0}, \quad \phi^{\prime}(0)=\phi^{0} .
$$

Aquí ,

$$
\langle A(t) \phi, w\rangle=a(t, \phi, w)=\int_{\Omega} a_{i j}(x, t) \frac{\partial \phi}{\partial x_{i}} \frac{\partial w}{\partial x_{j}} d x
$$

'Teorema 3.1. Sean

$$
\phi^{0} \in H_{0}^{1}(\Omega) \cap H^{2}(\Omega), \phi^{1} \in H_{0}^{1}(\Omega), f \in W^{1,1}\left(0, T ; L^{2}(\Omega)\right) .
$$

Entonces existe una única función $\phi: Q \rightarrow \mathbb{R}$ tal que

$$
\begin{array}{r}
\phi \in L^{\infty}\left(0, T ; H_{0}^{1}(\Omega) \cap H^{2}(\Omega)\right), \phi^{\prime} \in L^{\infty}\left(0, T ; H_{0}^{1}(\Omega)\right), \\
\phi^{\prime \prime} \in L^{\infty}\left(0, T ; L^{2}(\Omega)\right)
\end{array}
$$




$$
\begin{gathered}
\left(K_{1} \phi^{\prime}\right)^{\prime}-\left(K_{2} \phi\right)^{\prime}+K_{3} \phi+A(t) \phi=f \text { c.t.p. en } Q . \\
\phi(0)=\phi^{0}, \quad \phi^{\prime}(0)=\phi^{1} .
\end{gathered}
$$

El Teorema 3.1 se prueba aplicando el método de Faedo Galerkin con dos estimaciones. La solución $\phi$ obtenida en el teorema 3.1 se llama solución fuerte del problema homogéneo (3.1).

El Teorema 3.1 nos permite obtener el siguiente resultado:

Teorema 3.2. Sean

$$
\phi^{0} \in H_{0}^{1}(\Omega), \quad \phi^{1} \in L^{2}(\Omega), \quad f \in L^{1}\left(0, T ; L^{2}(\Omega)\right) .
$$

Entonces

Existe una única solución débil $\phi$ del problema (3.1) perteneciendo a la clase

$$
\phi \in C\left([0, T] ; H_{0}^{1}(\Omega)\right) \cap C^{1}\left([0, T] ; L^{2}(\Omega)\right) .
$$

La aplicación lineal

$$
\begin{gathered}
H_{0}^{1}(\Omega) \times L^{2}(\Omega) \times L^{1}\left(0, T ; L^{2}(\Omega)\right) \rightarrow C\left([0, T] ; H_{0}^{1}(\Omega)\right) \cap \\
C^{1}\left([0, T] ; L^{2}(\Omega)\right) \\
\left\{\phi^{0}, \phi^{1}, f\right\} \mapsto \phi
\end{gathered}
$$

es contínua, $\phi$ obtenida en (3.6).

La solución $\phi$ hallada en (3.6) satisface

$$
\frac{1}{2}\left|k_{1}^{1 / 2}(t) \phi^{\prime}(t)\right|^{2}+\frac{1}{2} a(t, \phi(t), \phi(t))=
$$




$$
\begin{aligned}
& =\frac{1}{2}\left|K_{1}^{1 / 2}(0) \phi^{1}\right|^{2}+\frac{1}{2} a\left(0, \phi^{0}, \phi^{0}\right)+ \\
& +\frac{1}{2} \int_{0}^{t} a^{\prime}(s, \phi(s), \phi(s)) d s-\frac{1}{2} \int_{0}^{t}\left(K_{1}^{\prime}(s) \phi^{\prime}(s), \phi^{\prime}(s)\right) d s+ \\
& +\int_{0}^{t}\left(K_{2}(s) \phi^{\prime}(s), \phi^{\prime}(s)\right) d s+\int_{0}^{t}\left(K_{2}^{\prime}(s) \phi(s), \phi^{\prime}(s)\right) d s- \\
& -\int_{0}^{t}\left(K_{3}(s) \phi(s), \phi^{\prime}(s)\right) d s+\int_{0}^{t}\left(f(s), \phi^{\prime}(s)\right) d s
\end{aligned}
$$

El Teorema 3.2 se prueba utilizando el teorema (3.1) mediante aproximaciones.

Ahora consideremos un problema homogéneo que será utilizado en el estudio de la regularidd de la solución $y=y(x, t)$ del problema (*) de la introducción. Esto es,

$$
\left\{\begin{array}{l}
\left(K_{1}(x, t) \phi^{\prime}\right)^{\prime}-\left(K_{2}(x, t) \phi\right)^{\prime}+K_{3}(x, t) \phi+A(t) \phi=f^{\prime} \text { en } Q \\
\phi=0 \text { sobre } \Sigma \\
\phi(0)=0, \phi^{\prime}(0)=0 \text { en } \Omega
\end{array}\right.
$$

Teorema 3.3. Sean

$$
f \in L^{2}\left(0, T ; H_{0}^{1}(\Omega)\right), f^{\prime} \in L^{2}\left(0, T ; L^{2}(\Omega)\right), f(0)=0 .
$$

Entonces la solución débil $\phi$ del problema (3.9) satisface 


$$
\begin{gathered}
\left|K_{1}^{1 / 2}(t) \phi^{\prime}(t)-K_{1}^{-1 / 2}(t) f(t)\right|+\|\phi(t)\| \leq \\
\quad \leq c \int_{0}^{T}\|f(t)\| d t, \quad \forall t \in[0, T]
\end{gathered}
$$

donde $c$ es una constante independiente de $\phi$ y $f$.

Demostración: La igualdad (3.8) del teorema 3.2 aplicada al problema (3.9) nos permite obtener:

$$
\begin{aligned}
& \frac{1}{2}\left|K_{1}^{1 / 2}(t) \phi^{\prime}(t)\right|^{2}+\frac{1}{2} a(t, \phi(t), \phi(t))= \\
& \frac{1}{2} \int_{0}^{t} a^{\prime}(s, \phi(s), \phi(s)) d s-\frac{1}{2} \int_{0}^{t}\left(K_{1}^{\prime}(s) \phi^{\prime}(s), \phi^{\prime}(s)\right) d s+ \\
& +\int_{0}^{t}\left(K_{2}(s) \phi^{\prime}(s), \phi^{\prime}(s)\right) d s+\int_{0}^{t}\left(K_{2}^{\prime}(s) \phi(s), \phi^{\prime}(s)\right) d s- \\
& -\int_{0}^{t}\left(K_{3}(s) \phi(s), \phi^{\prime}(s)\right) d s+\int_{0}^{t}\left(f^{\prime}(s), \phi^{\prime}(s)\right) d s .
\end{aligned}
$$

Integrando por partes y notando que $f(0)=0$, obtenemos:

$$
\begin{aligned}
& \int_{0}^{t}\left(f^{\prime}(s), \phi^{\prime}(s)\right) d s=\left(f(t), \phi^{\prime}(t)\right)+ \\
& +\int_{0}^{t}\left(K_{1}^{-1}(s) K_{1}^{\prime}(s) f(s), \phi^{\prime}(s)\right) d s- \\
& -\int_{0}^{t}\left(K_{1}^{-1}(s) f(s),\left(K_{1} \phi^{\prime}\right)^{\prime}(s)\right) d s
\end{aligned}
$$

sabemos que 


$$
\left(K_{1} \phi^{\prime}\right)^{\prime}=f^{\prime}-A(t) \phi+K_{2} \phi^{\prime}+K_{2}^{\prime} \phi-K_{3} \phi
$$

sustituyendo (3.13) en (3.12) resulta

$$
\begin{aligned}
& \int_{0}^{t}\left(f^{\prime}(s), \phi^{\prime}(s)\right) d s=\left(f(t), \phi^{\prime}(t)\right)+ \\
& +\int_{0}^{t}\left(K_{1}^{-1}(s) K_{1}^{\prime}(s) f(s), \phi^{\prime}(s)\right) d s-\int_{0}^{t}\left(K_{1}^{-1}(s) f(s), f^{\prime}(s)\right) d s+ \\
& \int_{0}^{t}\left\langle K_{1}^{-1}(s) f(s), A(s) \phi(s)\right\rangle d s-\int_{0}^{t}\left(K_{1}^{-1}(s) K_{2}(s) f(s), \phi^{\prime}(s)\right) d s- \\
& \int_{0}^{t}\left(K_{1}^{-1}(s) K_{2}^{\prime}(s) f(s), \phi(s) d s+\int_{0}^{t}\left(K_{1}^{-1}(s) K_{3}(s) f(s), \phi(s)\right) d s\right.
\end{aligned}
$$

después de integrar por partes el tercer término del lado derecho de la igualdad anterior, obtenemos:

$$
\begin{aligned}
& \int_{0}^{t}\left(f^{\prime}(s), \phi^{\prime}(s)\right) d s=\left(f(t), \phi^{\prime}(t)\right)-\frac{1}{2}\left(K_{1}^{-1}(t) f(t), f(t)\right)- \\
& -\frac{1}{2} \int_{0}^{t}\left(K_{1}^{-2}(s) K_{1}^{\prime}(s) f(s), f(s)\right) d s+\int_{0}^{t}\left(K_{1}^{-1}(s) K_{1}^{\prime}(s) f(s), \phi^{\prime}(s)\right) d s+ \\
& +\int_{0}^{t}\left\langle K_{1}^{-1}(s) f(s), A(s) \phi(s)\right\rangle d s-\int_{0}^{t}\left(K_{1}^{-1}(s) K_{2}(s) f(s), \phi^{\prime}(s)\right) d s- \\
& -\int_{0}^{t}\left(K_{1}^{-1}(s) K_{2}^{\prime}(s) f(s), \phi(s)\right) d s+\int_{0}^{t}\left(K_{1}^{-1}(s) K_{3}(s) f(s), \phi(s)\right) d s
\end{aligned}
$$


De las igualdades (3.11) y (3.14) se obtiene fácilmente:

$$
\begin{aligned}
& \frac{1}{2}\left|K_{1}^{1 / 2}(t) \phi^{\prime}(t)-K_{1}^{-1 / 2}(t) f(t)\right|^{2}+\frac{1}{2} a(t, \phi(t), \phi(t))= \\
& =\frac{1}{2} \int_{0}^{t} a^{\prime}(s, \phi(s), \phi(s)) d s-\frac{1}{2} \int_{0}^{t}\left(K_{1}^{\prime}(s) \phi^{\prime}(s), \phi^{\prime}(s)\right) d s+ \\
& +\int_{0}^{t}\left(K_{2}(s) \phi^{\prime}(s), \phi^{\prime}(s)\right) d s+\int_{0}^{t}\left(K_{2}^{\prime}(s) \phi(s), \phi^{\prime}(s)\right) d s- \\
& -\frac{1}{2} \int_{0}^{t}\left(K_{1}^{-2}(s) K_{1}^{\prime}(s) f(s), f(s)\right) d s+\int_{0}^{t}\left(K_{1}^{-1}(s) K_{1}^{\prime}(s) f(s), \phi^{\prime}(s)\right) d s+ \\
& +\int_{0}^{t}\left\langle K_{1}^{-1}(s) f(s), A(s) \phi(s)\right\rangle d s-\int_{0}^{t}\left(K_{1}^{-1}(s) K_{2}(s) f(s), \phi^{\prime}(s)\right) d s- \\
& -\int_{0}^{t}\left(K_{1}^{-1}(s) K_{2}^{\prime}(s) f(s), \phi(s)\right) d s+\int_{0}^{t}\left(K_{1}^{-1}(s) K_{3}(s) f(s), \phi(s)\right) d s .
\end{aligned}
$$

Haciendo $\theta=K_{1}^{1 / 2} \phi^{\prime}-K_{1}^{-1 / 2} f$ en (3.15) y sustituyendo $\phi^{\prime}$ por $K_{1}^{-1 / 2} \theta+$ $K_{1}^{-1} f$ en esta igualdad, obtenemos después de cálculos directos

$$
\begin{aligned}
& \frac{1}{2}|\theta(t)|^{2}+\frac{1}{2} a(t, \phi(t), \phi(t))= \\
& =\frac{1}{2} \int_{0}^{t} a^{\prime}(s, \phi(s), \phi(s)) d s-\frac{1}{2} \int_{0}^{t}\left(K_{1}^{-1}(s) K_{1}^{\prime}(s) \theta(s), \theta(s)\right) d s+ \\
& +\int_{0}^{t}\left(K_{1}^{-1}(s) K_{2}(s) \theta(s), \theta(s)\right) d s+\int_{0}^{t}\left(K_{1}^{-3 / 2}(s) K_{2}(s) f(s), \theta(s)\right) d s+
\end{aligned}
$$




$$
\begin{aligned}
& +\int_{0}^{t}\left(K_{1}^{-1 / 2}(s) K_{2}^{\prime}(s) \phi(s), \theta(s)\right)+\int_{0}^{t} a\left(s, K_{1}^{-1}(s) f(s), \phi(s)\right) d s+ \\
& +\int_{0}^{t}\left(K_{1}^{-1}(s) K_{3}(s) f(s), \phi(s)\right) d s .
\end{aligned}
$$

Mayorando la igualdad (3.16) deducimos

$$
\begin{gathered}
\frac{1}{2}|\theta(t)|^{2}+\frac{1}{2}\|\phi(t)\|^{2} \leq c \int_{0}^{t}\left(|\theta(s)|^{2}+\|\phi(s)\|^{2}\right) d s+ \\
+c \int_{0}^{t}\|f(s)\|(|\theta(s)|+\|\phi(s)\|) d s
\end{gathered}
$$

donde $c$ es una constante independiente de $\phi$ y $f$. Utilizando la desigualdad de Gronwall en la estimación (3.17) resulta

$$
|\theta(t)|+\|\phi(t)\| \leq c \int_{0}^{T}\|f(s)\| d s .
$$

Sustituyendo $\theta$ por su definición en (3.18), se deduce la estimación (3.10).

\section{DESIGUALDAD DIRECTA E INVERSA}

El objetivo de esta sección es estimar la derivada normal $\frac{\partial \phi}{\partial \nu}, \phi$ la solución débil del problema (3.1).

De acuerdo con la hipótesis (H4) tenemos

$$
a_{0} \xi_{i} \xi_{i} \leq a_{i j}(x, t) \xi_{i} \xi_{j} \leq a_{i} \xi_{i} \xi_{i}, \forall\{x, t\} \in \bar{Q}, \forall \xi \in \mathbb{R}^{\mathrm{n}}\left(\mathrm{a}_{0}>0\right)
$$

La energía del sistema (3.1) es

$$
E(t)=\frac{1}{2}\left|K_{1}^{1 / 2}(t) \phi^{\prime}(t)\right|^{2}+\frac{1}{2} a(t, \phi(t), \phi(t))
$$


en particular

$$
E_{0}=E(0)=\frac{1}{2}\left|K_{1}^{1 / 2}(0) \phi^{1}\right|^{2}+\frac{1}{2} a\left(0, \phi^{0}, \phi^{0}\right)
$$

Teorema 4.1. Sea $\phi$ la solución débil del problema (3.1). Entonces si $f=0$,

$$
E_{0} e^{-C_{0}} \leq E(t) \leq E_{0} e^{C_{0}}, \forall t \in[0, \infty[
$$

donde,

$$
\begin{aligned}
& C_{0}=a_{0}^{-1}\|\beta\|_{L^{1}(0,+\infty)}+\alpha_{0}^{-1}\left\|K_{1}^{\prime}\right\|_{L^{1}\left(0,+\infty, L^{\infty}(\Omega)\right)}+ \\
& +2 \alpha_{0}^{-1}\left\|K_{2}\right\|_{L^{1}\left(0,+\infty, L^{\infty}(\Omega)\right)}+a_{0}^{-1 / 2} \alpha_{0}^{-1 / 2} \lambda_{1}^{-1 / 2}\left\|K_{2}^{\prime}\right\|_{L^{1}\left(0,+\infty, L^{\infty}(\Omega)\right)}+ \\
& +a_{0}^{-1 / 2} \alpha_{0}^{-1 / 2} \lambda_{1}^{-1 / 2}\left\|K_{3}\right\|_{L^{1}\left(0,+\infty, L^{\infty}(\Omega)\right)} \\
& \beta(t)=\sup _{x \in \Omega}\left(\sum_{i, j=1}^{n}\left|a_{i j}^{\prime}(x, t)\right|^{2}\right)^{1 / 2} \in L^{1}(0,+\infty) .
\end{aligned}
$$

si $f \neq 0$,

$$
E(t) \leq c\left[E_{0}+\left(\int_{0}^{T}|f(t)|\right)^{2}\right], \quad \forall t \in[0, T]
$$

donde c es una constante independiente de la solución débil del problema (3.1).

Demostración. Probaremos la parte (4.3) del teorema 4.1. La parte (4.4) se obtiene de forma análoga a (4.3). Derivando con respecto a $t$ la igualdad (3.8) del teorema 3.2 , con $f=0$, y teniendo en cuenta (4.2) resulta: 


$$
\begin{aligned}
E^{\prime}(t)= & \frac{1}{2} a^{\prime}(t, \phi(t), \phi(t))-\frac{1}{2}\left(K_{1}^{\prime}(t) \phi^{\prime}(t), \phi^{\prime}(t)\right)+ \\
& +\left(K_{2}(t) \phi^{\prime}(t), \phi^{\prime}(t)\right)+\left(K_{2}^{\prime}(t) \phi(t), \phi^{\prime}(t)\right)- \\
& -\left(K_{3}(t) \phi(t), \phi^{\prime}(t)\right) .
\end{aligned}
$$

Mayorando el lado derecho de la igualdad anterior obtenemos

$$
\left|E^{\prime}(t)\right| \leq G(t) E(t)
$$

donde

$$
\begin{aligned}
& G(t)=a_{0}^{-1} \beta(t)+\alpha_{0}^{-1}\left\|K_{1}^{\prime}(t)\right\|_{L^{\infty}(\Omega)}+2 \alpha_{0}^{-1}\left\|K_{2}(t)\right\|_{L^{\infty}(\Omega)}+ \\
& a_{0}^{-1 / 2} a_{0}^{-1 / 2} \lambda_{1}^{-1 / 2}\left\|K_{2}^{\prime}(t)\right\|_{L^{\infty}(\Omega)}+a_{0}^{-1 / 2} \alpha_{0}^{-1 / 2} \lambda_{1}^{-1 / 2}\left\|K_{3}(t)\right\|_{L^{\infty}(\Omega)}
\end{aligned}
$$

de la desigualdad anterior se sigue:

$$
-G(t) E(t) \leq E^{\prime}(t) \leq G(t) E(t)
$$

Usando las hipótesis (H1)-(H4), sección 2, sobre las funciones $K_{1}$, $K_{2}, K_{3}$ y $a_{i j}$ podemos acotar cada término que define $G$ y de (4.5) se obtiene

$$
\int_{0}^{\infty} G(t) d t \leq C_{0}
$$

Combinando (4.6) y (4.7) concluimos la prueba del teorema.

Seguidamente, probaremos una igualdad que es fundamental en la obtención de la estimación para $\frac{\partial \phi}{\partial \nu}$ :

Teorema 4.2. Sea $\left(q_{\ell}\right)_{1 \leq \ell \leq n}$ un campo de vectores tal que $q_{\ell} \in$ $C^{1}(\bar{\Omega})$ para $1 \leq \ell \leq n$. Entonces cada solución débil $\phi$ del problema (3.1) verifica: 


$$
\begin{aligned}
& \frac{1}{2} \int_{\Sigma} a_{i j} \nu_{i} \nu_{j} q_{\ell} \nu_{\ell}\left(\frac{\partial \phi}{\partial \nu}\right)^{2} d \Sigma=\left.\left(K_{1} \phi^{\prime}, q_{\ell} \frac{\partial \phi}{\partial x_{\ell}}\right)\right|_{0} ^{T}+ \\
& +\frac{1}{2} \int_{Q} \frac{\partial\left(K_{1} q_{\ell}\right)}{\partial x_{\ell}} \phi^{2} d x d t-\frac{1}{2} \int_{Q} a_{i j} \frac{\partial \phi}{\partial x_{j}} \frac{\partial q_{\ell}}{\partial x_{\ell}} \frac{\partial \phi}{\partial x_{i}} d x d t+ \\
& +\int_{Q} a_{i j} \frac{\partial \phi}{\partial x_{j}} \frac{\partial q_{\ell}}{\partial x_{i}} \frac{\partial \phi}{\partial x_{\ell}} d x d t-\frac{1}{2} \int_{Q} \frac{\partial a_{i j}}{\partial x_{\ell}} \frac{\partial \phi}{\partial x_{j}} q_{\ell} \frac{\partial \phi}{\partial x_{i}} d x d t- \\
& -\int_{Q}\left(K_{2} \phi\right)^{\prime} q_{\ell} \frac{\partial \phi}{\partial x_{\ell}} d x d t-\int_{Q} K_{3} \phi q_{\ell} \frac{\partial \phi}{\partial x_{\ell}} d x d t- \\
& -\int_{Q} f q_{\ell} \frac{\partial \phi}{\partial x_{\ell}} d x d t .
\end{aligned}
$$

Demostración. Primero, probamos la igualdad (4.8) para soluciones fuertes $\phi$ del problema (3.1). Luego por densidad se sigue para soluciones débiles. Así $\phi(t) \in H_{0}^{1}(\Omega) \cap H^{2}(\Omega), \phi^{\prime}(t) \in H_{0}^{1}(\Omega)$.

Multiplicando la ecuación (3.1) 1 por $q \ell \frac{\partial \phi}{\partial x_{\ell}}$ e integrando sobre $Q$, obtenemos

$$
\begin{aligned}
& \int_{Q}\left(K_{1} \phi^{\prime}\right)^{\prime} q_{\ell} \frac{\partial \phi}{\partial x_{\ell}} d x d t-\int_{Q}\left(K_{2} \phi\right)^{\prime} q_{\ell} \frac{\partial \phi}{\partial x_{\ell}} d x d t+ \\
& +\int_{Q} K_{3} \phi q_{\ell} \frac{\partial \phi}{\partial x_{\ell}} d x d t+\int_{Q} A(t) \phi q_{\ell} \frac{\partial \phi}{\partial x_{\ell}} d x d t= \\
& =\int_{Q} f q_{\ell} \frac{\partial \phi}{\partial x_{\ell}} d x d t .
\end{aligned}
$$


Integrando por partes en $t$ y aplicando luego la fórmula de Green en el primer término de la expresión (4.9) resulta:

$$
\begin{gathered}
\int_{Q}\left(K_{1} \phi^{\prime}\right)^{\prime} q_{\ell} \frac{\partial \phi}{\partial x_{\ell}} d x d t=\left.\left(K_{1} \phi^{\prime}, q_{\ell} \frac{\partial \phi}{\partial x_{\ell}}\right)\right|_{0} ^{T}+ \\
+\frac{1}{2} \int_{Q} \frac{\partial\left(K_{1} q_{\ell}\right)}{\partial x_{\ell}}\left(K_{1} q_{\ell}\right) \phi^{\prime 2} d x d t
\end{gathered}
$$

Para el cuarto término de (4.9), usando la fórmula de Green se obtiene:

$$
\begin{aligned}
& \int_{Q} A(t) \phi q_{\ell} \frac{\partial \phi}{\partial x_{\ell}} d x d t=\int_{Q} a_{i j} \frac{\partial \phi}{\partial x_{j}} \frac{\partial q_{\ell}}{\partial x_{i}} \frac{\partial \phi}{\partial x_{\ell}} d x d t+ \\
& +\int_{Q} a_{i j} \frac{\partial \phi}{\partial x_{j}} q_{\ell} \frac{\partial^{2} \phi}{\partial x_{i} \partial x_{\ell}} d x d t-\int_{\Sigma} a_{i j} \frac{\partial \phi}{\partial x_{j}} q_{\ell} \frac{\partial \phi}{\partial x_{\ell}} \nu_{i} d \Sigma .
\end{aligned}
$$

Aplicando el operador $\frac{\partial}{\partial x_{\ell}}$ sobre $a_{i j} \frac{\partial \phi}{\partial x_{j}} q \frac{\partial \phi}{\partial x_{i}}$ y notando que $a_{i j}=a_{j i}$, deducimos usando la fórmula de Green en el segundo término del lado derecho de (4.11), que

$$
\begin{aligned}
& 2 \int_{Q} a_{i j} \frac{\partial \phi}{\partial x_{j}} q_{\ell} \frac{\partial^{2} \phi}{\partial x_{i} \partial x_{\ell}} d x d t= \\
& =-\int_{Q} \frac{\partial a_{i j}}{\partial x_{\ell}} \frac{\partial \phi}{\partial x_{j}} q_{\ell} \frac{\partial \phi}{\partial x_{i}} d x d t- \\
& -\int_{Q} a_{i j} \frac{\partial \phi}{\partial x_{j}} \frac{\partial q_{\ell}}{\partial x_{\ell}} \frac{\partial \phi}{\partial x_{i}} d x d t+\int_{\Sigma} a_{i j} \frac{\partial \phi}{\partial x_{j}} q_{\ell} \frac{\partial \phi}{\partial x_{i}} \nu_{\ell} d \Sigma .
\end{aligned}
$$

Se sabe que $\frac{\partial \phi}{\partial x_{i}}=\nu_{i} \frac{\partial \phi}{\partial \nu}$ sobre $\Gamma$ (Ver J.L. Lions [9]), por lo tanto 


$$
\begin{aligned}
& \frac{1}{2} \int_{\sigma} a_{i j} \frac{\partial \phi}{\partial x_{j}} q_{\ell} \frac{\partial \phi}{\partial x_{i}} \nu_{\ell} d \Sigma-\int_{\Sigma} a_{i j} \frac{\partial \phi}{\partial x_{j}} q_{\ell} \frac{\partial \phi}{\partial x_{\ell}} \nu_{i} d \Sigma= \\
& -\frac{1}{2} \int_{\Sigma} a_{i j} \nu_{i} \nu_{j} q_{\ell} \nu_{\ell}\left(\frac{\partial \phi}{\partial \nu}\right)^{2} d \Sigma .
\end{aligned}
$$

Combinando las expresiones (4.11), (4.12) y (4.13) se obtiene:

$$
\begin{aligned}
& \int_{Q} A(t) \phi q_{\ell} \frac{\partial \phi}{\partial x_{\ell}} d x d t=\int_{Q} a_{i j} \frac{\partial \phi}{\partial x_{j}} \frac{\partial q_{\ell}}{\partial x_{i}} \frac{\partial \phi}{\partial x_{\ell}} d x d t- \\
& -\frac{1}{2} \int_{Q} \frac{\partial a_{i j}}{\partial x_{\ell}} \frac{\partial \phi}{\partial x_{j}} q_{\ell} \frac{\partial \phi}{\partial x_{i}} d x d t-\frac{1}{2} \int_{Q} a_{i j} \frac{\partial \phi}{\partial x_{j}} \frac{\partial q_{\ell}}{\partial x_{\ell}} \frac{\partial \phi}{\partial x_{i}} d x d t- \\
& -\frac{1}{2} \int_{\Sigma} a_{i j} \nu_{i} \nu_{j} q_{\ell} \nu_{\ell}\left(\frac{\partial \phi}{\partial \nu}\right)^{2} d \Sigma .
\end{aligned}
$$

Sustituyendo (4.10) y (4.14) en (4.9) y transponiendo términos adecuadamente, resulta la identidad (4.8).

La siguiente desigualdad a demostrar se llama desigualdad directa para el problema (3.1).

Teorema 4.3. Sea $\phi$ la solución débil del problema (3.1). Entonces $\frac{\partial \phi}{\partial \nu} \in L^{2}(\Sigma) y$

$$
\int_{\Sigma}\left(\frac{\partial \phi}{\partial \nu}\right)^{2} d \Sigma \leq c\left[E_{0}+\left(\int_{0}^{T}|f(t)| d t\right)^{2}\right]
$$

donde $c$ es una constante independiente de $\phi$.

Demostración: Del teorema (4.1) parte (4.4) se tiene la siguiente estimación 


$$
\left|K_{1}^{1 / 2}(t) \phi^{\prime}(t)\right|^{2}+a(t,, \phi(t), \phi(t)) \leq c E_{0}+c\left(\int_{0}^{T}|f(t)| d t\right)^{2}, \forall t \in[0, T]
$$

Consideremos la identidad (4.8) con $q_{\ell}=\nu_{\ell}$ sobre $\Gamma$. Observemos que, usando la estimación (4.15), Is integrales sobre $\Omega$ de (4.8) pueden ser acotadas por

$$
c\left[E_{0}+\left(\int_{0}^{T}|f(t)| d t\right)^{2}\right]
$$

las integrales sobre $Q$ por

$$
c(T+1)\left[E_{0}+\left(\int_{0}^{T}|f(t)| d t\right)^{2}\right]
$$

y la integral $\int_{Q} f q_{\ell} \frac{\partial_{\phi}}{\partial} d x d t$ por

$$
c E_{0}^{1 / 2} \int_{0}^{T}|f(t)| d t+c\left(\int_{0}^{T}|f(t)| d t\right)^{2}
$$

Por otro lado

$$
\frac{1}{2} \int_{\Sigma} a_{i j} \nu_{i} \nu_{j} \nu_{\ell} \nu_{\ell}\left(\frac{\partial \phi}{\partial \nu}\right)^{2} d \Sigma \geq \frac{1}{2} a_{0} \int_{\Sigma}\left(\frac{\partial \phi}{\partial \nu}\right)^{2} d \Sigma .
$$

Combinando las estimaciones anteriores y (4.8) con $q_{\ell}=\nu_{\ell}$ sobre $\Gamma$ resulta:

$$
\int_{\Sigma}\left(\frac{\partial \phi}{\partial \nu}\right)^{2} d \Sigma \leq c(T+1)\left[E_{0}+\left(\int_{0}^{T}|f(t)| d t\right)^{2}\right]+c E_{0}^{1 / 2} \int_{0}^{T}|f(t)| d t
$$

Mayorando la desigualdad anterior se obtiene el resultado el teorema 4.3 .

Para establecer la desigualdad inversa para el problema 3.1 con $f=0$ introducimos algunas notaciones. Del Teorema 4.1 parte (4.3), obtenemos 


$$
\begin{gathered}
C_{1} E_{0} \leq E(t) \leq C_{2} E_{0}, \quad \forall t \in[0, \infty[ \\
\left(C_{1}=e^{-C_{0}}, \quad C_{2}=e^{C_{0}}\right)
\end{gathered}
$$

El tiempo $T_{0}$ es definido por:

$$
\begin{aligned}
T_{0}= & {\left[2 a_{0}^{-1 / 2}\left\|\left.K_{1}\right|_{L^{\infty}(Q)} ^{1 / 2} R\left(x^{0}\right)+\alpha_{0}^{-1}\right\| \eta \|_{L^{1}(0,+\infty)} R\left(x^{0}\right)+\right.} \\
& \left.+M_{1}+M_{2}+M_{3}\right] C_{2} C_{1}^{-1} \delta^{-1}
\end{aligned}
$$

donde

$$
\begin{gathered}
\eta(t)=\sup _{\Omega} \operatorname{ess}\left(\sum_{\ell=1}^{n}\left|\frac{\partial K_{1}}{\partial x_{\ell}}(x, t)\right|^{2}\right)^{1 / 2} \in L^{1}(0,+\infty) \\
M_{1}=\frac{(n-\delta)}{2} \alpha_{0}^{-1 / 2} a_{0}^{-1} \lambda_{1}^{-1}\left[a_{0}^{1 / 2} \lambda_{1}^{1 / 2}\left\|K_{2}\right\|_{L^{1}\left(0,+\infty, L^{\infty}(\Omega)\right)}\right. \\
\left.\quad+2 \alpha_{0}^{1 / 2}\left\|K_{2}^{\prime}\right\|_{L^{1}\left(0,+\infty, L^{\infty}(\Omega)\right)}\right] \\
M_{2}=\alpha_{0}^{-1 / 2} a_{0}^{-1} \lambda_{1}^{-1} R\left(x^{0}\right)\left[a_{0}^{1 / 2} \lambda_{i} \mid K_{2} \|_{L^{1}\left(0,+\infty, L^{\infty}(\Omega)\right)}\right. \\
\quad+2 \alpha_{0}^{1 / 2} \lambda_{1}^{1 / 2}\left\|K_{2}^{\prime}\right\|_{\left.L^{1}\left(0,+\infty, L^{\infty}(\Omega)\right)\right]} \\
M_{3}=\lambda_{1}^{-1} a_{0}^{-1}\left\|K_{3}\right\|_{L^{1}\left(0,+\infty, L^{\infty}(\Omega)\right)}\left[(n-\delta)+2 \lambda_{1}^{1 / 2} R\left(x^{0}\right)\right]
\end{gathered}
$$


Teorema 4.4. Sea $T>T_{0}$. Entonces cada solución débil $\phi$ del problema (3.1) con $f=0$, verifica

$$
\frac{1}{2} R\left(x^{0}\right) a_{1} \int_{\Sigma\left(x^{0}\right)}\left(\frac{\partial \phi}{\partial \nu}\right)^{2} d \Sigma \geq \delta C_{1}\left(T-T_{0}\right) E_{0}
$$

Obsevación 4.1. Notemos que si las. funciones $K_{1}=1, K_{2}=$ $K_{3}=0$ y $a_{i j}=\delta_{i j}$ entonces $C_{1}=C_{2}=\delta=a_{0}=1, M_{1}=M_{2}=\eta=0$ lo que implica $T_{0}=2 R\left(x^{0}\right)$. Este es el tiempo $T_{0}$ determinado en J.L. Lions [9] y en V. Komornik [7] para la ecuación $u^{\prime \prime}-\Delta u=0$.

Demostración del teorema 4.4.: Consideremos la identidad (4.8) del teorema 4.2 con el campo particular $q_{\ell}=m_{\ell}=x_{\ell}-x_{\ell}^{0} \mathrm{y}$ después de hacer cálculos directos obtenemos

$$
\begin{aligned}
& \frac{1}{2} \int_{\Sigma} a_{i j} \nu_{i} \nu_{j} m_{\ell} \nu_{\ell}\left(\frac{\partial \phi}{\partial \nu}\right)^{2} d \Sigma= \\
& =\left.\left(K_{1} \phi^{\prime}, m_{\ell} \frac{\partial \phi}{\partial x_{\ell}}+\frac{n-\delta}{2} \phi\right)\right|_{0} ^{T}+\delta \int_{0}^{T} E(t) d t+ \\
& +\frac{1}{2} \int_{Q} \frac{\partial K_{1}}{\partial x_{\ell}} m_{\ell} \phi^{\prime 2} d x d t+ \\
& +\int_{Q}\left[(1-\delta) a_{i j} \frac{\partial \phi}{\partial x_{i}} \frac{\partial \phi}{\partial x_{j}}-\frac{1}{2} \frac{\partial a_{i j}}{\partial x_{\ell}} m_{\ell} \frac{\partial \phi}{\partial x_{i}} \frac{\partial \phi}{\partial x_{j}}\right] d x d t- \\
& -\frac{n-\delta}{2} \int_{Q}\left(K_{2} \phi\right)^{\prime} \phi d x d t-\int_{Q}\left(K_{2} \phi\right)^{\prime} m_{\ell} \frac{\partial \phi}{\partial x_{\ell}} d x d t+ \\
& +\frac{n-\delta}{2} \int_{Q} K_{3} \phi^{2} d x d t+\int_{Q} K_{3} \phi m_{\ell} \frac{\partial \phi}{\partial x_{\ell}} d x d t
\end{aligned}
$$


donde $\delta$ es un número real positivo arbitrario.

Utilizando la hipótesis $(H 4)_{5}$ en la identidad (4.18) obtenemos la siguiente desigualdad

$$
\begin{aligned}
& \frac{1}{2} \int_{\Sigma} a_{i j} \nu_{i} \nu_{j} m_{\ell} \nu_{\ell}\left(\frac{\partial \phi}{\partial \nu}\right)^{2} d \Sigma \geq \\
& \geq\left.\left(K_{1} \phi^{\prime}, m_{\ell} \frac{\partial \phi}{\partial x_{\ell}}+\frac{n-\delta}{2} \phi\right)\right|_{0} ^{T}+\delta \int_{0}^{T} E(t) d t+ \\
& \frac{1}{2} \int_{Q} \frac{\partial K_{1}}{\partial x_{\ell}} m_{\ell} \phi^{\prime 2} d x d t-\frac{n-\delta}{2} \int_{Q}\left(K_{2} \phi\right)^{\prime} \phi d x d t- \\
& -\int_{Q}\left(K_{2} \phi\right)^{\prime} m_{\ell} \frac{\partial \phi}{\partial x_{\ell}} d x d t+\frac{n-\delta}{2} \int_{Q} K_{3} \phi^{2} d x d t+ \\
& +\int_{Q} K_{3} \phi m_{\ell} \frac{\partial \phi}{\partial x_{\ell}} d x d t .
\end{aligned}
$$

Haciendo cálculos análogos a los realizados en J.L. Lions [8] y [9]

$$
\begin{gathered}
\left|\left(K_{1} \phi^{\prime}, m_{\ell} \frac{\partial \phi}{\partial x_{\ell}}+\frac{n-\delta}{2} \phi\right)\right| \leq \\
\leq\left\|K_{1}\right\|_{L^{\infty}(Q)}^{1 / 2}\left[\frac{\mu}{2}\left|K_{1}^{1 / 2} \phi^{\prime}\right|^{2}+\frac{1}{2 \mu}\left|m_{\ell} \frac{\partial \phi}{\partial x_{\ell}}+\frac{n-\delta}{2} \phi\right|^{2}\right] .
\end{gathered}
$$

Tomando $\mu=a_{0}^{-1 / 2} R\left(x^{0}\right)>0$ y utilizando la estimación (4.16) en la desigualdad anterior, resulta:

$$
\left|\left(K_{1} \phi^{\prime}, m_{\ell} \frac{\partial \phi}{\partial x_{\ell}}+\frac{n-\delta}{2} \phi\right)\right| \leq a_{0}^{-1 / 2}\left\|K_{1}\right\|_{L^{\infty}(Q)}^{1 / 2} R\left(x^{0}\right) C_{2} E_{0}
$$


lo que implica

$$
\left.\left(K_{1} \phi^{\prime}, m_{\ell} \frac{\partial \phi}{\partial x_{\ell}}+\frac{n-\delta}{2} \phi\right)\right|_{0} ^{T} \geq 2 a_{0}^{-1 / 2}\left\|K_{1}\right\|_{L^{\infty}(Q)}^{1 / 2} R\left(x^{0}\right) C_{2} E_{0}
$$

Los otros términos del miembro de la derecha de (4.19) quedan acotadas utilizando la estimación (4.16) de la siguiente manera:

$$
\begin{gathered}
\delta \int_{0}^{T} E(t) d t \geq \delta T C_{1} E_{0} . \\
\frac{1}{2} \int_{Q} \frac{\partial K_{1}}{\partial x_{\ell}} m_{\ell} \phi^{2} d x d t \geq-\alpha_{0}^{-1}\|\eta\|_{L^{1}(0,+\infty)} R\left(x^{0}\right) C_{2} E_{0} . \\
-\frac{n-\delta}{2} \int_{Q}\left(K_{2} \phi\right)^{\prime} \phi d x d t \geq \\
\geq-\frac{n-\delta}{2} \alpha_{0}^{-1 / 2} a_{0}^{-1 / 2} \lambda_{1}^{-1 / 2}\left\|K_{2}\right\|_{L^{1}\left(0,+\infty, L^{\infty}(\Omega)\right)} C_{2} E_{0}- \\
-(n-\delta) a_{0}^{-1} \lambda_{1}^{-1}\left\|K_{2}^{\prime}\right\|_{L^{1}\left(0,+\infty, L^{\infty}(\Omega)\right)} C_{2} E_{0} . \\
-\int_{Q}\left(K_{2} \phi\right)^{\prime} m_{\ell} \frac{\partial \phi}{\partial x_{\ell}} d x d t \geq \\
\geq-\alpha_{0}^{-1 / 2} a_{0}^{-1 / 2} R\left(x^{0}\right)\left\|K_{2}\right\|_{L^{1}\left(0,+\infty, L^{\infty}(\Omega)\right)} C_{2} E_{0}- \\
-2 a_{0}^{-1} \lambda_{1}^{-1 / 2} R\left(x^{0}\right)\left\|K_{2}^{\prime}\right\|_{L^{1}\left(0,+\infty, L^{\infty}(\Omega)\right)} C_{2} E_{0} .
\end{gathered}
$$




$$
\begin{aligned}
& \frac{n-\delta}{2} \int_{Q} K_{3} \phi^{2} d x d t \geq-\frac{n-\delta}{2} a_{0}^{-1} \lambda_{1}^{-1}\left\|K_{3}\right\|_{L^{1}\left(0,+\infty, L^{\infty}(\Omega)\right)} C_{2} E_{0} \\
& \int_{Q} K_{3} \phi m_{\ell} \frac{\partial \phi}{\partial x_{\ell}} d x d t \geq-2 a_{0}^{-1} \lambda_{1}^{-1 / 2} R\left(x^{0}\right)\left\|K_{3}\right\|_{L^{1}\left(0,+\infty, L^{\infty}(\Omega)\right)} C_{2} E_{0}
\end{aligned}
$$

Así, utilizando las estimaciones (4.20)-(4.26) en la desigualdad (4.19) obtenemos:

$$
\frac{1}{2} \int_{\Sigma\left(x^{a}\right)} a_{i j} \nu_{i} \nu_{j} \nu_{\ell} m_{\ell}\left(\frac{\partial \phi}{\partial \nu}\right)^{2} d \Sigma \geq \delta C_{1}\left(T-\dot{T}_{0}\right) E_{0}
$$

El miembro de la izquierda de (4.27) se puede acotar como en J.L. Lions [9] por

$$
\frac{1}{2} R\left(x^{0}\right) a_{1} \int_{\Sigma\left(x^{0}\right)}\left(\frac{\partial \phi}{\partial \nu}\right)^{2} d \Sigma .
$$

Combinando (4.27) y (4.28) finalizamos la prueba del teorema.

\section{CONTROLABILIDAD EXACTA}

En esta sección concluiremos la prueba del teorema 2.1. Consideremos el problema

$$
\left\{\begin{array}{l}
\left(K_{1}(x, t) z^{\prime}\right)^{\prime}+K_{2}(x, t) z^{\prime}+K_{3}(x, t) z+A(t) z=0 \text { en } Q \\
z=v \text { sobre } \Sigma \\
z(0)=z^{0}, z^{\prime}(0)=z^{1} \text { en } \Omega
\end{array}\right.
$$

Primero definiremos el concepto de solución del problema 5.1. Multiplicando ambos miembros de $(5.1)_{1}$ por $\theta=\theta(x, t)$ e integrando por partes sobre $Q$ formalmente, resulta: 


$$
\begin{aligned}
& \int_{Q}\left[\left(K_{1} z^{\prime}\right)^{\prime}+K_{2} z^{\prime}+K_{3} z+A(t) z\right] \theta d x d t= \\
& =-\int_{\Omega} K_{1}(0) z^{\prime}(0) \theta(0) d x+\int_{\Omega} K_{1}(0) z(0) \theta^{\prime}(0) d x- \\
& -\int_{\Omega} K_{2}(0) z(0) \theta(0) d x+\int_{\Sigma} a_{i j} \nu_{i} \nu_{j} \frac{\partial \theta}{\partial \nu} z d \Sigma+ \\
& +\int_{Q} z f d x d t
\end{aligned}
$$

donde $\theta$ es la solución del problema

$$
\left\{\begin{array}{l}
\left(K_{1} \theta^{\prime}\right)^{\prime}-\left(K_{2} \theta\right)^{\prime}+K_{3} \theta+A(t) \theta=f \text { en } Q \\
\theta=0 \text { sobre } \Sigma \\
\theta(T)=\theta^{\prime}(T)=0
\end{array}\right.
$$

Si $f \in L^{1}\left(0, T ; L^{2}(\Omega)\right)$, por el teorema 3.2 y gracias a la reversibilidad del problema (5.3) con respecto a la variable tiempo sobre el intervalo $[0, T]$, tenemos que la solución $\theta$ del problema (5.3) verifica

$$
\begin{aligned}
& \theta \in C\left([0, T] ; H_{0}^{1}(\Omega)\right) \cap C^{1}\left([0, T] ; L^{2}(\Omega)\right) \\
& \left|\theta^{\prime}(0)\right|+\|\theta(0)\| \leq c \int_{0}^{T}|f(t)| d t
\end{aligned}
$$

y por el teorema 4.3 y reversibilidad del tiempo se tiene

$$
\frac{\partial \theta}{\partial \nu} \in L^{2}(\Sigma), \quad\left\|\frac{\partial \theta}{\partial \nu}\right\|_{L^{2}(\Sigma)} \leq c \int_{0}^{T}|f(t)| d t
$$

donde $c$ es una constante independiente de $\phi$ y $f$.

Motivados por (5.2)-(5.5) introducimos la siguiente definición: Sea 


$$
z^{0} \in L^{2}(\Omega), z^{1} \in H^{-1}(\Omega), v \in L^{2}(\Sigma) .
$$

Decimos que $z \in L^{\infty}\left(0, T ; L^{2}(\Omega)\right)$ es una solución ultradébil definida por transposición del problema (5.1) con datos en la clase (5.6) si

$$
\begin{aligned}
& \int_{0}^{T}(z, f) d t=\left\langle K_{1}(0) z^{1}, \theta(0)\right\rangle-\left(K_{1}(0) z^{0}, \theta^{\prime}(0)\right)+ \\
& +\left(K_{2}(0) z^{0}, \theta(0)\right)-\int_{0}^{T}\left(v, a_{i j} \nu_{i} \nu_{j} \frac{\partial \theta}{\partial \nu}\right)_{L^{2}(\Gamma)} d t
\end{aligned}
$$

para cada $f \in L^{1}\left(0, T ; L^{2}(\Omega)\right)$ donde $\theta$ está relacionado con $f$ por el problema (5.3).

Claramente la solución $z$ dada anteriormente es única. También tenemos, de (5.4) y (5.5), que

$$
\|z\|_{L^{\infty}\left(0, T ; L^{2}(\Omega)\right)} \leq c\left(\left|z^{0}\right|+\left\|z^{1}\right\|_{H^{-1}(\Omega)}+\|v\|_{L^{2}(\Sigma)}\right),
$$

donde $c$ es una constante independiente de $z$.

Seguidamente probaremos un resultado que utilizaremos para demostrar la regularidad de la solución ultradébil del problema (5.1).

Sea $f \in \mathcal{D}(Q), \mathcal{D}(Q)$ es el espacio de las funciones de prueba sobre $Q$, y $\theta$ la solución débil del problema

$$
\left\{\begin{array}{l}
\left.\left(K_{(} x, t\right) \theta^{\prime}\right)^{\prime}-\left(K_{2}(x, t) \theta\right)^{\prime}+K_{3}(x, t) \theta+A(t) \theta=f^{\prime} \text { en } Q \\
\theta=0 \text { sobre } \Sigma \\
\theta(0)=0, \theta^{\prime}(0)=0 \text { en } \Omega .
\end{array}\right.
$$

Del teorema 3.3 (Vea sección 3), tenemos:

$$
\left|K_{1}^{1 / 2}(t) \theta^{\prime}(t)-K_{1}^{-1 / 2}(t) f(t)\right|+\|\theta(t)\| \leq c \int_{0}^{T}\|f(t)\| d t, \forall t \in[0, T]
$$


donde $c$ es una constante independiente de $\theta$ y $f$. En virtud del teorema 4.3, $\frac{\partial \theta}{\partial \nu} \in L^{2}(\Sigma)$.

Lema 5.1. Cada solución débil $\theta$ del problema (5.9) verifica:

$$
\left\|\frac{\partial \theta}{\partial \nu}\right\|_{L^{2}(\Sigma)} \leq c \int_{0}^{T}\|f(t)\| d t
$$

donde $c$ es una constante independiente de $\theta$ y $f$.

Demostración: Utilizando la identidad (4.8) del teorema 4.2 para el problema (5.9) y las siguientes igualdades

$$
\begin{gathered}
\theta^{\prime}=K_{1}^{-1 / 2}\left(K_{1}^{1 / 2} \theta^{\prime}-K_{1}^{-1 / 2} f\right)+K_{1}^{-1} f \\
\theta^{\prime 2}=K_{1}^{-1}\left(K_{1}^{1 / 2} \theta^{\prime}-K_{1}^{-1 / 2} f\right)^{2}+2 K_{1}^{-3 / 2} f\left(K_{1}^{1 / 2} \theta^{\prime}-K_{1}^{-1 / 2} f\right)+ \\
\quad+K_{1}^{-2} f^{2}
\end{gathered}
$$

después de cálculos directos se obtiene:

$$
\begin{aligned}
& \frac{1}{2} \int_{\Sigma} a_{i j} \nu_{i} \nu_{j} q_{\ell} \nu_{\ell}\left(\frac{\partial \theta}{\partial \nu}\right)^{2} d \Sigma=\left.\left(K_{1} \theta^{\prime}, q_{\ell} \frac{\partial \theta}{\partial x_{\ell}}\right)\right|_{0} ^{T}+ \\
& +\frac{1}{2} \int_{Q} K_{1}^{-1} \frac{\partial\left(K_{1} q_{\ell}\right)}{\partial x_{\ell}}\left(K_{1}^{1 / 2} \theta^{\prime}-K_{1}^{-1 / 2} f\right)^{2} d x d t+ \\
& +\int_{Q} K_{1}^{-3 / 2} \frac{\partial K_{1}}{\partial x_{\ell}} q_{\ell} f\left(K_{1}^{1 / 2} \theta^{\prime}-K_{1}^{-1 / 2} f\right) d x d t- \\
& -\int_{Q} K_{1}^{-1 / 2} \frac{\partial f}{\partial x_{\ell}} q_{\ell}\left(K_{1}^{1 / 2} \theta^{\prime}-K_{1}^{-1 / 2} f\right) d x d t-
\end{aligned}
$$




$$
\begin{aligned}
& \text { Controlsbilidad Exacta de la Ecuación del Telégrafo Generalizada } \\
& -\frac{1}{2} \int_{Q} a_{i j} \frac{\partial \theta}{\partial x_{j}} \frac{\partial q_{\ell}}{\partial x_{\ell}} \frac{\partial \theta}{\partial x_{i}} d x d t+ \\
& +\int_{Q} a_{i j} \frac{\partial \theta}{\partial x_{j}} \frac{\partial q_{\ell}}{\partial x_{i}} \frac{\partial \theta}{\partial x_{\ell}} d x d t-\frac{1}{2} \int_{Q} \frac{\partial a_{i j}}{\partial x_{\ell}} \frac{\partial \theta}{\partial x_{j}} q_{\ell} \frac{\partial \theta}{\partial x_{i}} d x d t- \\
& -\int_{Q} K_{2}^{\prime} q_{\ell} \frac{\partial \theta}{\partial x_{\ell}} \theta d x d t- \\
& -\int_{Q} K_{1}^{-1 / 2} K_{2} q_{\ell} \frac{\partial \theta}{\partial x_{\ell}}\left(K_{1}^{1 / 2} \theta^{\prime}-K_{1}^{-1 / 2} f\right) d x d t- \\
& -\int_{Q} K_{1}^{-1} K_{2} q_{\ell} \frac{\partial \theta}{\partial x_{\ell}} f d x d t+\int_{Q} K_{3} q_{\ell} \theta \frac{\partial \theta}{\partial x_{\ell}} d x d t .
\end{aligned}
$$

Tomando $q_{\ell} \in C^{1}(\bar{\Omega}), 1 \leq \ell \leq n$ tal que $q_{\ell} \nu_{\ell}=1$ sobre $\Gamma$, y acotando la expresión (5.11) por la estimación (5.10) obtenemos una constante $c>0$ tal que

$$
\frac{1}{2} \int_{\Sigma} a_{i j} \nu_{i} \nu_{j}\left(\frac{\partial \theta}{\partial \nu}\right)^{2} d \Sigma \leq c\left(\int_{0}^{T}\|f(t)\| d t\right)^{2}
$$

lo que implica, por la hipótesis de coercividad sobre las funciones $a_{i j}$,

$$
\left\|\frac{\partial \theta}{\partial \nu}\right\|_{L^{2}(\Sigma)} \leq c \int_{0}^{T}\|f(t)\| d t
$$

donde $c$ es una constante independiente de $\theta$ y $f$.

Teorema 5.1. Cada solución ultradébil $z$ del problema (5.1) tiene la regularidad

$$
z \in C\left([0, T] ; L^{2}(\Omega)\right) \cap C^{1}\left([0, T] ; H^{-1}(\Omega)\right)
$$

y la aplicación lineal 


$$
\begin{aligned}
L^{2}(\Omega) \times H^{-1}(\Omega) \times L^{2}(\Sigma) & \rightarrow C\left([0, T] ; L^{2}(\Omega)\right) \cap C^{1}\left([0, T] ; H^{-1}(\Omega)\right) \\
\left\{z^{0}, z^{1}, v\right\} & \mapsto z
\end{aligned}
$$

es contínua.

Demostración: Primero probemos que $z \in C\left([0, T] ; L^{2}(\Omega)\right)$. Siendo $z^{0}, z^{1}, v$ pertenecientes a la clase (5.6) existen sucesiones de vectores $\left(z_{\mu}^{0}\right),\left(z_{\mu}^{1}\right),\left(v_{\mu}\right)$ de $H_{0}^{1}(\Omega), L^{2}(\Omega), H_{0}^{2}\left(0, T ; H^{3 / 2}(\Gamma)\right)$, respectivamente, tal que

$$
\begin{aligned}
& z_{\mu}^{0} \rightarrow z^{0} \text { en } L^{2}(\Omega), z_{\mu}^{1} \rightarrow z^{1} \text { en } H^{-1}(\Omega), \\
& v_{\mu} \rightarrow v \text { en } L^{2}(\Sigma) .
\end{aligned}
$$

Sea $\widehat{v}_{\mu}$ una función en $H_{0}^{2}\left(0, T ; H^{2}(\Omega)\right)$ tal que $\gamma \widehat{v}_{\mu}=\left\{v_{\mu}, 0\right\}, \gamma$ función trazo sobre $\Gamma$, y $u_{\mu}$ la solución del problema

$$
\left\{\begin{array}{l}
\left(K_{1} u_{\mu}^{\prime}\right)^{\prime}+K_{2} u_{\mu}^{\prime}+K_{3} u_{\mu}+A(t) u_{\mu}= \\
=-\left[\left(K_{1} \widehat{v}_{\mu}^{\prime}\right)^{\prime}+K_{2} \widehat{v}_{\mu}^{\prime}+K_{3} \widehat{v}_{\mu}+A(t) \widehat{v}_{\mu}\right] \text { en } Q \\
u_{\mu}=0 \text { sobre } \Sigma \\
u_{\mu}(0)=z_{\mu}^{0}, u_{\mu}^{\prime}(0)=z_{\mu}^{1} \text { en } \Omega
\end{array}\right.
$$

Entonces se demuestra de manera análoga al teorema 3.2 que la solución débil $u_{\mu}$ del problema (5.14) tiene la regularidad

$$
u_{\mu} \in C\left(\left[0, T ; H_{0}^{1}(\Omega)\right) \cap C^{1}\left([0, T] ; L^{2}(\Omega)\right)\right.
$$

Luego tenemos que $z_{\mu}=u_{\mu}+\widehat{v}_{\mu}$ es la solución del problema (5.1) con datos $z_{\mu}^{0}, z_{\mu}^{\mathrm{1}}, v_{\mu}$ y $z_{\mu} \in C\left([0, T] ; L^{2}(\Omega)\right)$. Por lo tanto, de (5.8)

$\left\|z_{\mu}-z\right\|_{L^{\infty}\left(0, T ; L^{2}(\Omega)\right)} \leq c\left[\left|z_{\mu}^{0}-z^{0}\right|+\left\|z_{\mu}^{1}-z^{1}\right\|_{H^{-1}(\Omega)}+\left\|v_{\mu}-v\right\|_{L^{2}(\Sigma)}\right]$. 
Tomando límite en esta expresión y usando la convergencia (5.13) y la regularidad de $z_{\mu}$, obtenemos $z \in C\left([0, T] ; L^{2}(\Omega)\right)$.

Ahora consideremos $f \in \mathcal{D}(Q)$ y $\theta$ la solución débil del problema

$$
\left\{\begin{array}{l}
\left(K_{1} \theta^{\prime}\right)^{\prime}-\left(K_{2} \theta\right)^{\prime}+K_{3} \theta+A(t) \theta=f^{\prime} \text { en } Q \\
\theta=0 \text { sobre } \Sigma \\
\theta(T)=0, \theta^{\prime}(T)=0
\end{array}\right.
$$

Entonces por la reversibilidad del problema anterior y el teorema 3.3 obtenemos que

$$
\left|K_{1}^{1 / 2}(t) \theta^{\prime}(t)-K_{1}^{-1 / 2}(t) f(t)\right|+\|\theta(t)\| \leq c \int_{0}^{T}\|f(t)\| d t, \forall t \in[0, T]
$$

y por el lema 5.1

$$
\left\|\left.\frac{\partial \theta}{\partial \nu}\right|_{L^{2}(\Sigma)} \leq c \int_{0}^{T}\right\| f(t) \| d t .
$$

Tenemos que $z^{\prime} \in H^{-1}\left(0, T ; L^{2}(\Omega)\right)$, ya que $z \in L^{2}\left(0, T ; L^{2}(\Omega)\right)$. Entonces

$$
\left\langle z^{\prime}, f\right\rangle=-\left(z, f^{\prime}\right)_{L^{2}(Q)}=-\int_{0}^{T}\left(z, f^{\prime}\right) d t
$$

Como $z$ es una solución ultradébil definida por transposición del problema (5.1) tenemos de (5.15)

$$
\begin{aligned}
& \int_{0}^{T}\left(z, f^{\prime}\right) d t=\left\langle K_{1}(0) z^{1}, \theta(0)\right\rangle-\left(K_{1}(0) z^{0}, \theta^{\prime}(0)\right)+ \\
& +\left(K_{2}(0) z^{0}, \theta(0)\right)-\int_{0}^{T}\left(v, a_{i j} \nu_{i} \nu_{j} \frac{\partial \theta}{\partial \nu}\right)_{L^{2}(\Gamma)} d t
\end{aligned}
$$


De las estimaciones (5.16) y (5.17) obtenemos

$$
\left|\left\langle z^{\prime}, f\right)\right| \leq c\left[\left|z^{0}\right|+\left\|z^{1}\right\|_{H^{-1}(\Omega)}+\|v\|_{L^{2}(\Sigma)}\right] \int_{0}^{T}\|f(t)\| d t, \forall f \in \mathcal{D}(Q)
$$

lo que implica por densidad de $\mathcal{D}(Q)$ en $L^{1}\left(0, T ; H_{0}^{1}(\Omega)\right)$ que $z^{\prime} \in L^{\infty}(0$, $\left.T ; H^{-1}(\Omega)\right)$ y

$$
\| z^{\prime} \mid L_{L^{\infty}\left(0, T ; H^{-1}(\Omega)\right)} \leq c\left[\left|z^{0}\right|+\left\|z^{1}\right\|_{H^{-1}(\Omega)}+\|\left. v\right|_{L^{z}(\Sigma)}\right] .
$$

Por argumentos similares como en la primera parte de la prueba del teorema y observando que $z_{\mu}^{\prime} \in C\left([0, T] ; H^{-1}(\Omega)\right)$ concluimos que $z^{\prime} \in$ $C\left([0, T] ; H^{-1}(\Omega)\right)$.

La continuidad de la aplicación lineal $\left\{z^{0}, z^{1}, v\right\} \mapsto z$ se obtiene por (5.8) y (5.18).

Ahora finalizaremos la prueba del teorema 2.1. Sea $\phi$ la solución débil del problema

$$
\left\{\begin{array}{l}
\left(K_{1} \phi^{\prime}\right)^{\prime}-\left(K_{2} \phi\right)^{\prime}+K_{3} \phi+A(t) \phi=0 \text { en } Q^{\circ} \\
\phi=0 \text { sobre } \Sigma \\
\phi(0)=\phi^{0}, \phi^{\prime}(0)=\phi^{1} \text { en } \Omega
\end{array}\right.
$$

Con $\left\{\phi^{0}, \phi^{1}\right\} \in H_{0}^{1}(\Omega) \times L^{2}(\Omega)$. Entonces debido a los teoremas $3.2,4.3$ $\mathrm{y}(4.4)$ tenemos

$$
\phi \in C\left([0, T] ; H_{0}^{1}(\Omega)\right) \cap C^{1}\left([0, T] ; L^{2}(\Omega)\right)
$$

$\frac{\partial \phi}{\partial \nu} \in L^{2}(\Sigma) \mathrm{y}$

$$
\frac{2 \delta C_{1}}{R\left(x^{0}\right) a_{1}}\left(T-T_{0}\right) E_{0} \leq\left\|\frac{\partial \phi}{\partial \nu}\right\|_{L^{2}\left(\Sigma\left(x^{0}\right)\right)} \leq c E_{0} .
$$

Con $\phi$ construimos la solución ultradébil $\psi$ del problema 


$$
\left\{\begin{array}{l}
\left(K_{1} \psi^{\prime}\right)^{\prime}+K_{2} \psi^{\prime}+K_{3} \psi+A(t) \psi=0 \text { en } Q \\
\psi=\left\{\begin{array}{c}
\frac{\partial \phi}{\partial \nu} \text { sobre } \Sigma\left(x^{0}\right) \\
0 \text { sobre } \Sigma \backslash \Sigma\left(x^{0}\right) \\
\psi(T)=0, \psi^{\prime}(T)=0 \text { en } \Omega
\end{array}\right.
\end{array}\right.
$$

Entonces por el teorema 5.1 y gracias a la reversibilidad con respecto al tiempo en $[0, T]$ del problema (5.21), tenemos que $\psi$ pertenece a la clase

$$
\psi \in C\left([0, T] ; L^{2}(\Omega)\right) \cap C^{1}\left([0, T] ; H^{-1}(\Omega)\right) .
$$

También tenemos

$$
\begin{gathered}
\left\langle K_{1}(0) \psi^{\prime}(0)+K_{2}(0) \psi(0), \phi^{0}\right\rangle-\left(K_{1}(0) \psi(0), \phi^{1}\right)= \\
\int_{\Sigma\left(x^{0}\right)} a_{i j} \nu_{i} \nu_{j}\left(\frac{\partial \phi}{\partial \nu}\right)^{2} d \Sigma
\end{gathered}
$$

La expresión (5.22) induce a definir el siguiente operador

$$
\begin{aligned}
& A: H_{0}^{1}(\Omega) \times L^{2}(\Omega) \rightarrow H^{-1}(\Omega) \times L^{2}(\Omega) \\
& \Lambda\left\{\phi^{0}, \phi^{1}\right\}=\left\{K_{1}(0) \psi^{\prime}(0)+K_{2}(0) \psi(0),-K_{1}(0) \psi(0)\right\}
\end{aligned}
$$

De (5.22), obtenemos

$$
a_{0}\left\|\frac{\partial \phi}{\partial \nu}\right\|_{L^{2}\left(\Sigma\left(x^{0}\right)\right)}^{2} \leq\left\langle\Lambda\left\{\phi^{0}, \phi^{1}\right\},\left\{\phi^{0}, \phi^{1}\right\}\right\rangle \leq a_{1}\left\|\frac{\partial \phi}{\partial \nu}\right\|_{L^{2}\left(\Sigma\left(x^{0}\right)\right)}
$$

De la estimación anterior y (5.21) implica que $\Lambda$ es inyectiva. Con $\left\{\widehat{\phi}^{0}, \widehat{\phi}^{1}\right\}$ determinamos la solución débil del problema (5.19) y con $\frac{\partial \widehat{\phi}}{\partial v}$, la solución ultradébil definida por transposición $\widehat{\psi}$ del problema (5.21). 
Analogamente a la expresión (5.22) se obtienen las siguientes expresiones:

$$
\begin{aligned}
& \left\langle\Lambda\left\{\phi^{0}, \phi^{1}\right\},\left\{\widehat{\phi}^{0}, \widehat{\phi}^{1}\right\}\right\rangle=\int_{\Sigma\left(x^{0}\right)} a_{i j} \nu_{i} \nu_{j} \frac{\partial \widehat{\phi}}{\partial \nu} \frac{\partial \phi}{\partial \nu} d \Sigma \\
& \left\langle\Lambda\left\{\widehat{\phi}^{0}, \widehat{\phi}^{1}\right\},\left\{\phi^{0}, \phi^{1}\right\}\right\rangle=\int_{\Sigma\left(x^{0}\right)} a_{i j} \nu_{i} \nu_{j} \frac{\partial \phi}{\partial \nu} \frac{\partial \widehat{\phi}}{\partial \nu} d \Sigma
\end{aligned}
$$

De estas dos últimas igualdades obtenemos que $\Lambda$ es auto-adjunto. Así

$\Lambda$ es un isomorfismo de $H_{0}^{1}(\Omega) \times L^{2}(\Omega)$ en $H^{-1} \times L^{2}(\Omega)$.

Sea $\left\{y^{0}, y^{1}\right\} \in L^{2}(\Omega) \times H^{-1}(\Omega)$. Entonces por (5.23), existe $\left\{\phi^{0}, \phi^{1}\right\} \in H_{0}^{1}(\Omega) \times L^{2}(\Omega)$ tal que

$$
\Lambda\left\{\phi^{0}, \phi^{1}\right\}=\left\{K_{1}(0) y^{1}+K_{2}(0) y^{0},-K_{1}(0) y^{0}\right\} .
$$

De esta igualdad y de la definición del operador $\Lambda$ concluimos

$$
\psi(0)=y^{0}, \quad \psi^{\prime}(0)=y^{1}
$$

donde $\psi$ es la única solución ultradébil de (5.21) y $\phi$ es la única solución débil de (5.19).

Si consideramos

$$
v=\frac{\partial \phi}{\partial \nu} \text { en } \Sigma\left(x^{0}\right)
$$

en el problema (2.1) con datos iniciales $\left\{y^{0}, y^{1}\right\}$ perteneciendo a $L^{2}(\Omega) \times$ $H^{-1}(\Omega)$ resulta que tal problema tiene una única solución ultradébil $y$. Observemos que de (5.21) y (5.24) obtenemos que $\psi$ es también solución ultradébil del problema (2.1). Luego por la unicidad de solución vemos que $y=\psi$ y consecuentemente de $(5.21)_{3}$ concluimos que

$$
y(T)=0, \quad y^{\prime}(T)=0 .
$$


Agradecimientos. El autor expresa su más sincera gratitud a la valiosa colaboración y orientación de los profesores L.A. Medeiros y M. Milla Miranda en relación con este trabajo.

\section{Bibliografía}

[1] Adams, R., Sobolev Spaces, Academic Press, New York, 1975.

[2] Brezis, H., Operateurs Maximaux Monotones et semigroups de contractions dans les spaces de Hilbert, North Holland Publishing Co., Amsterdam, 1973.

[3] Brezis, H., Analyse fonctionnelle: théorie et applications, Masson, Paris, 1983.

[4] Fabre, C., Comportement au voisinage du bord de quelques équations d'évolution linéaire. Thèse de Doctorat de l'université Pierre et Marie Curie, Paris 1990.

[5] Fabre, C. and Puel, J.P., Behavior near the boundary for solutions of the wave equation, Journal of Differential Equations 106, pp. 186-213 (1993).

[6] Fuentes, R., Exact Controllability for Temporally Wave Equation, Portugaliae Mathematica, Vol. 51 Fas. 4, pp. $475-488$ (1994).

[7] Komornik, V., Exact controllability in short time for the wave equation, Analyse Non Linéaire, Ann. Inst. Henri Poincaré, 6 (1989), pp. 153-164.

[8] Lions, J.L., Exact controllability, stabilization and perturbations for distributed systems, SIAM Review, 30 (1988), pp. 1-68.

[9] Lions, J.L., Contrôlabilité Exacte, Stabilisation de Systèmes Distribuées, Tome 1, Masson RMA8, 1988.

[10] Lions J.L., Contrôlabilité Exacte, Stabilisation de Systèmes Distribuées, Tome 2, Masson RMA9, 1988.

[11] Lions, J.L. and Magenes, E., Problèmes aux Limites Non Homogènes et Applications, Vol 1, Dunod, 1968. 
[12] Maciel. A.B., On Hyperbolic-Parabolic Equation with a Continuous Nonlinearity; Nonlinear Analysis, Theory, Methods \& Applications, Vol. 20, no. 6, pp. $745-754,1993$.

[13] Medeiros, L.A. and Fuentes, R., Exact controllability for a model of the one dimensional elasticity, 36 Seminário Brasileiro de Análise, 1992.

[14] Medeiros, L.A., Exact controllability for a Timoshenko model of vibrations of beams. Advances in Mathematical Sciences and Applications, $\mathrm{n}^{\circ} 1$, Vol. 2, 1993 (Japan).

[15] Milla Miranda, M., Contrôlabilité exacte de l'équation des ondes dans des domaines non cylindriques, C.R. Acad. Sci. Paris, t. 317, Série 1, p. 495-499, 1993.

[16] Milla Miranda, M., HUM and the wave equation with variable coefficients (To appear).

[17] Muñoz Rivera, J.E., Exact controllability: coefficient depending on the time, SIAM J. Control and Optimization, 28 (1990), pp. 498-501.

[18] Puel, J.P., Contrôlabilité Exacte et Comportement au Voisinage du Bord des Solutions de l'Équations de Ondes, Lectures at IM-UFRJ, Rio de Janeiro, RJ. Brasil, 1991.

[19] Tijonov, A.N. and Samarsky, A.A., Ecuaciones de la Física Matemática, Editorial Mir (Moscu), 1972.

[20] Zuazua, E., Controlabilidad exacta y estabilización de la ecuación de ondas, Textos de Métodos Matemáticos, 23 IM-UFRJ, Rio de Janeiro, RJ, Brasil, 1990. 
[21] Zuazua, E., An introduction to the exact controllability for distributed systems, CMAF, Universidade de Lisboa, Portugal, 1990.

Departamento de Matemática

Recibido: 20 de Abril de 1994

\section{Universidad Estatal de Maringá}

CP 331 - CEP 87020 - MARINGA PR

Brazil 